\title{
ac Josephson Effect in Finite-Length Nanowire Junctions with Majorana Modes
}

\author{
Pablo San-Jose, ${ }^{1}$ Elsa Prada, ${ }^{2}$ and Ramón Aguado ${ }^{2}$ \\ ${ }^{1}$ Instituto de Estructura de la Materia (IEM-CSIC), Serrano 123, 28006 Madrid, Spain \\ ${ }^{2}$ Instituto de Ciencia de Materiales de Madrid (ICMM-CSIC), Cantoblanco, 28049 Madrid, Spain
}

(Received 22 February 2012; published 18 June 2012)

\begin{abstract}
It has been predicted that superconducting junctions made with topological nanowires hosting Majorana bound states (MBS) exhibit an anomalous $4 \pi$-periodic Josephson effect. Finding an experimental setup with these unconventional properties poses, however, a serious challenge: for finite-length wires, the equilibrium supercurrents are always $2 \pi$ periodic as anticrossings of states with the same fermionic parity are possible. We show, however, that the anomaly survives in the transient regime of the ac Josephson effect. Transients are, moreover, protected against decay by quasiparticle poisoning as a consequence of the quantum Zeno effect, which fixes the parity of Majorana qubits. The resulting longlived ac Josephson transients may be effectively used to detect MBS.
\end{abstract}

DOI: 10.1103/PhysRevLett.108.257001

PACS numbers: 74.50.+r, 03.65.Vf, 73.21. Hb

Recently, it has been argued that Majorana bound states (MBS) should appear in topological insulators [1] and semiconductors with strong spin-orbit (SO) coupling [2-5] which, in proximity to $s$-wave superconductors, may behave as topological superconductors (TS). MBS in these TS can be understood as Bogoliubov-de Gennes (BdG) quasiparticles appearing inside the superconducting gap, exactly at zero energy (for a review, see Ref. [6]). The TS phase is tunable, which has spurred a great deal of experimental activity toward detecting MBS. Tunneling through such TS is expected to show a zero-bias anomaly signaling the presence of MBS [7-13]. However, such anomaly, which has been recently observed [14], only proves a necessary condition. A more stringent test can be established by measuring the Josephson effect through a junction between two TS nanowires. Kitaev predicted [15] that such a Josephson effect has an anomalous $4 \pi$ periodicity in the superconducting (SC) phase difference $\phi \equiv$ $\phi_{1}-\phi_{2}$ between the two wires. This fractional Josephson current $I_{J} \sim \sin \left(\frac{\phi}{2}\right)[4,5,15-20]$ can be understood in terms of fermion parity (FP): if FP is preserved, there is a protected crossing of Majorana states at $\phi=\pi$ [21] with perfect population inversion, namely the system cannot remain in the ground state as $\phi$ evolves from 0 to $2 \pi$ adiabatically, unlike for standard Andreev bound states (ABS) [22]. However, any finite length of the two TS regions gives rise to two additional MBS at the wire ends (which are connected to topologically trivial regions), allowing for the hybridization of two states of the same FP. This results in residual splittings at $\phi=\pi$ which, despite being exponentially small, destroy the fractional effect as the system remains in the ground state for all $\phi$ [24].

The $4 \pi$ periodicity can be restored in two ways. On the one hand, one may employ a fully topological circuit [25], which is free of additional MBS. In other words, by employing an $S^{\prime} N S^{\prime}$ geometry (where $S^{\prime}$ is a TS and $N$ is a normal, nontopological region), as opposed to an
$S S^{\prime} N S^{\prime} S$ junction (where $S$ is a topologically trivial superconductor). Unfortunately, this is presently a difficult experimental challenge using nanowires. Alternatively, the $4 \pi$ Josephson effect may be recovered by biasing the junction and thereby sweeping $\phi$ fast enough through the anticrossing such that Landau-Zener (LZ) processes [26] induce nonadiabatic transitions between states of the same FP. By the same token, however, transitions to the continuum of states above the gap and, hence, parity mixing processes will be unavoidable [27].

The above hindrances pose relevant questions. Is the Josephson effect a useful tool to detect MBS in realistic TS wires? Do finite TS length and quasiparticle poisoning inevitably destroy the fractional periodicity? We here prove that the ac currents in the TS phase contain anomalously long-lived $4 \pi$-periodic transients which are tunable through both bias and gate voltages. Thus, the Josephson effect may still be exploited to provide unequivocal proof of the existence of MBS in finite-length TS. Interestingly, the duration of the long $4 \pi$-periodic transients may be increased by the well-known, though somewhat counterintuitive, quantum Zeno effect, whereby a strong coupling to a decohering environment helps to confine the dynamics of a quantum system into a desired sector of the Hilbert space [28].

Low-energy description.-Consider a semiconducting one-dimensional wire with chemical potential $\mu_{S^{\prime}}$, SO coupling $\alpha$, and Zeeman splitting $\mathcal{B}$ (given by $\mathcal{B}=g \mu_{B} B / 2$, where $B$ is an in-plane magnetic field, $\mu_{B}$ is the Bohr magneton and $g$ is the wire $g$-factor). When the wire is proximity coupled to an $s$-wave superconductor of gap $\Delta_{S}$, a SC pairing term $\Delta_{S^{\prime}}<\Delta_{S}$ may be induced. The wire is then arranged into a Josephson device as illustrated in Fig. 1(a). The resulting hybrid system SS'NS'S can be driven into a TS phase where two MBS are formed at the ends of each $S^{\prime}$ region, denoted by $\gamma_{1,2}$ and $\gamma_{3,4}$, when $\mathcal{B}>\mathcal{B}_{c} \equiv$ $\sqrt{\mu_{S^{\prime}}^{2}+\Delta_{S^{\prime}}^{2}}[4,5]$. The low-energy Majorana sector appears 

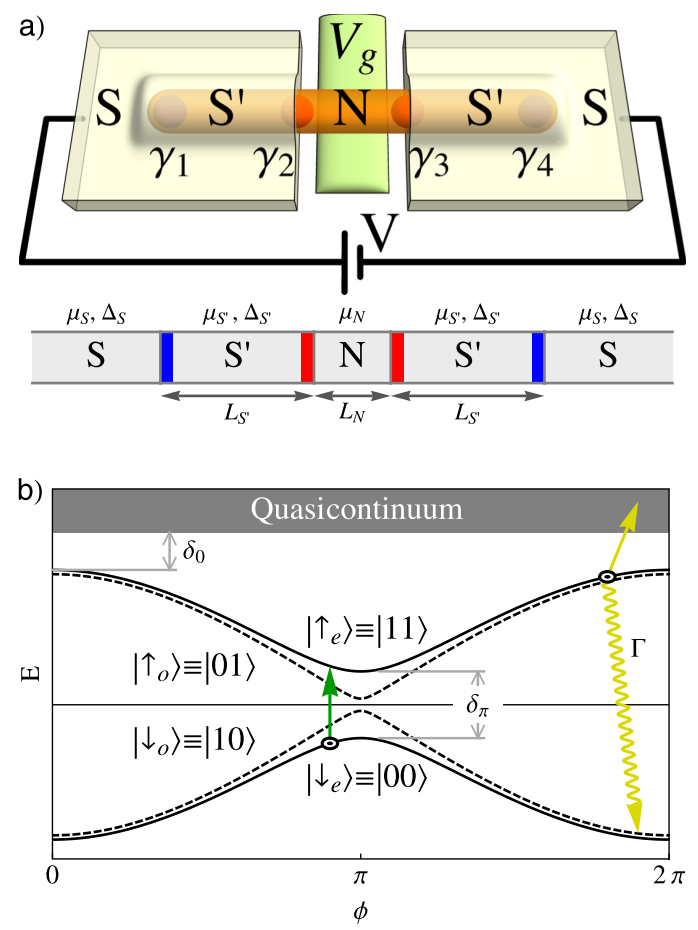

FIG. 1 (color online). (a) Schematics: A nanowire of length $L=L_{S^{\prime}}+L_{N}+L_{S^{\prime}}$ in contact with two $s$-wave superconductors (with gap $\Delta_{S}$ ), develops a proximity-induced SC gap $\Delta_{S^{\prime}}<\Delta_{S}$ and four Majorana modes $\gamma_{1,2,3,4}$. The transparency of the junction can be controlled by a gate voltage $V_{g}$. (b) Energy of the four lowest many-body states in the TS phase, as a function of SC phase difference $\phi$ across $N$. They correspond to even (solid) and odd (dashed) fillings of the two lowest ABS, see Fig. 2(b). Note the avoided crossing of size $\delta_{\pi}$ at $\phi=\pi$ and the detachment gap $\delta_{0}$ at $\phi=0$.

for energies $\varepsilon<\Delta_{\text {eff }}$, where $\Delta_{\text {eff }}$ is the effective SC gap [Fig. 2(b)].

Isolated MBS $\gamma_{i}$ are zero-energy superpositions of a particle and a hole. A pair of Majoranas $\gamma_{1,2}$ may be fused into a Dirac fermion $c^{\dagger}=\left(\gamma_{1}-i \gamma_{2}\right) / \sqrt{2}$, such that the operator $2 c^{\dagger} c-1=2 i \gamma_{1} \gamma_{2}$ with eigenvalues -1 and 1 in states $|0\rangle$ and $|1\rangle=c^{\dagger}|0\rangle$ defines FP [29]. Thus, a spatial overlap of two MBS hybridizes them into eigenstates of opposite FP. In a TS wire of length $L_{S^{\prime}}$, the decay distance of the two MBS pinned at the wire ends is the effective coherence length $\xi_{\text {eff }}=\hbar v_{F} / \pi \Delta_{\text {eff }}$. Their overlap will

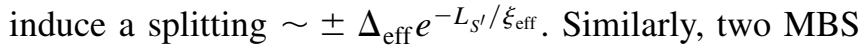
at either side of a Josephson junction with phase difference $\phi$ and transparency $T$ will hybridize into even or odd fermion states with energies $\sim \pm \Delta_{\text {eff }} \sqrt{T} \cos \phi / 2$, [16,17,27]. In the setup of Fig. 1, four MBS (two "inner" $\gamma_{2,3}$ and two "outer" $\gamma_{1,4}$ ) hybridize both through the Josephson junction (region $N$ ) and the finite length $S^{\prime}$ regions. The resulting eigenstates are empty and filled states of two Dirac fermions $d_{1,2}^{\dagger}(\phi)$, constructed as two $\phi$-dependent (orthogonal) superpositions of the two fermions $c_{\text {in }}^{\dagger}=\left(\gamma_{2}+i \gamma_{3}\right) / \sqrt{2}$ and $c_{\text {out }}^{\dagger}=\left(\gamma_{1}+i \gamma_{4}\right) / \sqrt{2}$,
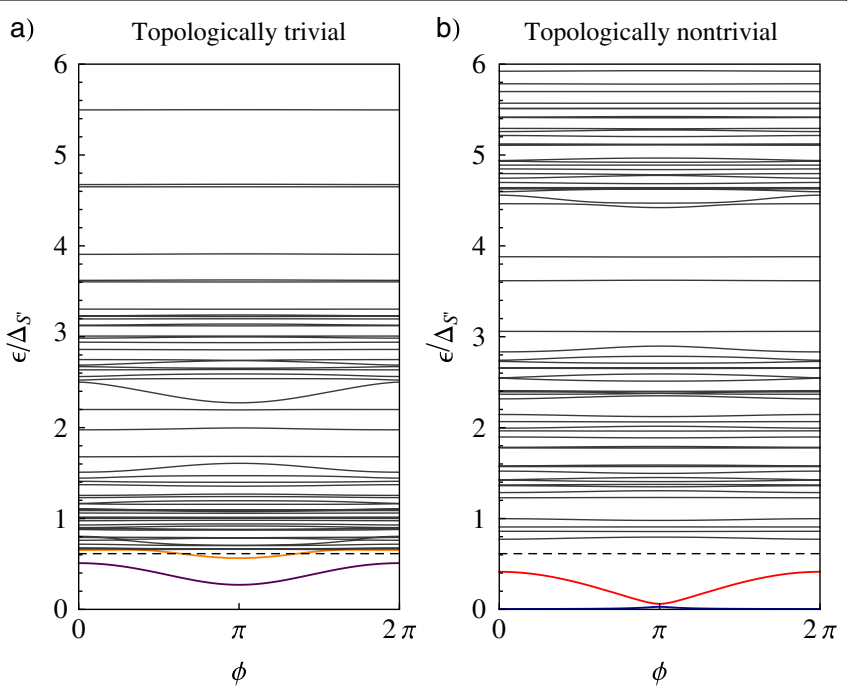

FIG. 2 (color online). Andreev bound states (ABS) for a SS'NS'S junction with normal conductance $G=0.72 G_{0}$ (with $\left.G_{0}=2 e^{2} / h\right)$, an induced gap $\Delta_{S^{\prime}}=218 \mu \mathrm{eV}$, and wire's length of $4 \mu \mathrm{m}$ (where $l_{s o}=216 \mathrm{~nm}$ for a InSb wire). In the topologically trivial phase (a), $\mathcal{B}=0.36 \mathrm{meV}=0.5 \mathcal{B}_{c}$, whereas in the nontrivial phase $(\mathrm{b}), \mathcal{B}=1.1 \mathrm{meV}=1.5 \mathcal{B}_{c}$. The dashed line denotes the wire effective gap $\Delta_{\text {eff }}=130 \mu \mathrm{eV}$ that separates localized ABS from the quasicontinuum.

which are themselves obtained from the fusion of the inner and outer MBS, respectively. We denote eigenenergies by $E_{n_{1} n_{2}}$ and eigenstates as $\left|n_{1} n_{2}\right\rangle$, where $n_{1}, n_{2}=0,1$ are the occupations of fermions $d_{1}^{\dagger}$ and $d_{2}^{\dagger}$. Two of them have even total parity, $\left|\downarrow_{e}\right\rangle \equiv|00\rangle,\left|\uparrow_{e}\right\rangle \equiv|11\rangle=d_{2}^{\dagger} d_{1}^{\dagger}|00\rangle$, and the other two are odd, $\left|\downarrow_{o}\right\rangle \equiv|10\rangle=d_{1}^{\dagger}|00\rangle$, $\left|\uparrow_{o}\right\rangle \equiv|01\rangle=$ $d_{2}^{\dagger}|00\rangle$ [33]. $E_{n_{1} n_{2}}(\phi)$ anti-cross at $\phi=\pi$ within sameparity sectors [Fig. 1(b)] and, hence, the supercurrents are $2 \pi$ periodic.

A $4 \pi$-periodic Josephson effect can, nevertheless, be recovered by inducing LZ transitions with a voltage bias, such as $\left|\downarrow_{e}\right\rangle \rightarrow\left|\uparrow_{e}\right\rangle$ [green arrow in Fig. 1(b)] [34]. To describe the response of a realistic biased junction, however, an extension of the simplified Majorana model above is required. Indeed, nonadiabatic driving may also induce inelastic transitions into delocalized states above the TS gap [yellow arrow in Fig. 1(b)]. These latter transitions induce an effective parity-mixing rate (yellow wiggly arrow) which couples even and odd sectors (quasiparticle poisoning). A proper description of such dynamics involves a calculation of all the Andreev levels (both below and above $\Delta_{\text {eff }}$ ) coupled to the continuum (above $\Delta_{S}$ ) of the junction, which we develop in what follows.

Andreev levels. - The full spectrum of single-particle eigenstates may be obtained by diagonalizing the $\mathrm{BdG}$ equations for the geometry in Fig. 1(a) [23]. We obtain $H_{\mathrm{BdG}}=\frac{1}{2} \sum_{n}\left(d_{n}^{\dagger} d_{n}-d_{n} d_{n}^{\dagger}\right) \varepsilon_{n}$, with eigenenergies $\varepsilon_{n}(\phi)$ plotted in Figs. 2(a) and 2(b) for a representative junction. The corresponding single particle excitations, called Andreev bound states (ABS), are defined as 
$|n(\phi)\rangle=d_{n}^{\dagger}(\phi)|\Omega(\phi)\rangle$, with $|\Omega(\phi)\rangle$ denoting the ground state. In the short junction limit, $L_{N} \ll \xi_{\text {eff }}$, only two ABS lie below the effective gap $\Delta_{\text {eff }}$. Upon crossing into the TS phase, these two levels, initially localized around the $N$ region in the nontopological phase [purple and orange (bottom) curves in Fig. 2(a)], reconnect into the two $d_{1,2}^{\dagger}(\phi)$ Majorana branches. The lowest Majorana branch [blue (bottom) curve in Fig. 2(b), level $d_{1}^{\dagger}$ ] has a weak $\phi$ energy dependence, and is dominated by the outer MBS ( $c_{\text {out }}^{\dagger}$ fermion, $\varepsilon_{1} \approx \Delta_{\text {eff }} e^{-L_{s^{\prime}} / \xi_{s^{\prime}}}$ ), whereas the one at higher energy [red (second from bottom) curve, $d_{2}^{\dagger}$ ] results mostly from the fusion of the inner MBS $\left(c_{\text {in }}^{\dagger}\right.$ fermion, $\varepsilon_{2} \approx \Delta_{\text {eff }} \sqrt{T} \cos \phi / 2$ ).

Due to the finite length $L_{S^{\prime}}>\xi_{S^{\prime}}$, a dense set (quasicontinuum) of ABS delocalized across the TS wire appears within a large energy window $\Delta_{S}>\varepsilon>\Delta_{\text {eff }}$ above the low-energy sector. This quasicontinuum (which approaches a continuum as $L_{S^{\prime}} \rightarrow \infty$ ), separates the Majorana branches from the true continuum of the problem at $\varepsilon>\Delta_{S}$. Importantly, for finite transparency junctions, the Majorana levels detach from the quasicontinuum, as opposed to the topologically trivial case or a standard SNS junction [35], where the ABS touch the continuum at $\phi=0,2 \pi$. We have verified that the detachment, denoted by $\delta_{0}$ in Fig. 1(b), increases as the transmission $T$ of the normal region becomes smaller, $\delta_{0} / \Delta_{\text {eff }} \approx 1-\sqrt{T}$, in agreement with simpler models [16,17,27].

ac Josephson effect.-We now study the Josephson current across the junction when biased with a voltage $V$. The bias makes the phase difference $\phi$ time dependent, $\phi(t)=2 \mathrm{eV} t / \hbar=\omega_{J} t$, where $\omega_{J}$ is the Josephson frequency. We calculate the Josephson current through the biased junction in terms of a reduced density matrix in the Majorana sector. Notably, its evolution contains paritymixing terms as a result of the Andreev levels above $\Delta_{\text {eff }}$ that have been traced out [23]. The goal is to identify smoking-gun features in $I(t)$, in the form of fractional frequency components, that may unambiguously determine the existence of MBS in the TS phase. It has been shown, however, that in the presence of finite dissipation (e.g., nonzero parity mixing), the current becomes, in the long time limit, strictly $2 \pi$ periodic, as a consequence of Floquet theorem, and that, as a result, nothing remains in the stationary current to qualitatively distinguish the trivial from the TS phase [27]. The two phases, however, exhibit crucial differences in their spectra that give rise to very different features in their transient regimes, $I\left(t<t_{T}\right)$. The spectral features of the TS phase (sharp $\phi=\pi$ anticrossing and detachment of the Majorana sector from the quasicontinuum in the TS phase, see Fig. 2) allows the even and odd Majorana subspaces in the TS phase to evolve almost coherently performing $4 \pi$-periodic population oscillations during a potentially long transient time $t_{T} \gg T_{2 \pi}$, where $T_{2 \pi}=2 \pi / \omega_{J}$ is the period of the Josephson driving. We now characterize the transient regime in detail. At bias $2 \mathrm{eV}<\delta_{\pi}$, the junction will remain in the initial ground state $|00\rangle$, resulting in an adiabatic $2 \pi$-periodic current [blue curve (top) in Fig. 3(c)]. Increasing $2 \mathrm{eV}$ above $\delta_{\pi}$ induces LZ transitions at $\phi=\pi$ from $\left|\downarrow_{e}\right\rangle=|00\rangle$ into $\left|\uparrow_{e}\right\rangle=|11\rangle$, which tend to produce a perfect population inversion as $2 \mathrm{eV} \gg \delta_{\pi}$. Then, by virtue of the finite detachment $\delta_{0}$, if $V$ remains smaller than a certain $V_{\text {MAR }}$ (quantified below), the population escape into the quasicontinuum as $\phi$ crosses $2 \pi$ will be negligible, and the junction will subsequently re-invert into its ground state $\left|\downarrow_{e}\right\rangle$ at $\phi=3 \pi$. The repetition of this inversion process gives rise to a transient $4 \pi$-periodic Josephson current $I \sim \sin \phi / 2$ [red curve (middle) in Fig. 3(c)], with a superimposed
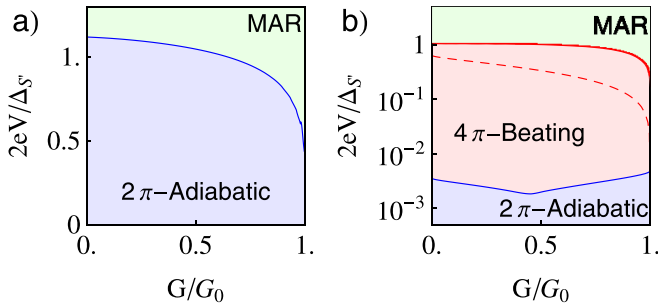

c)
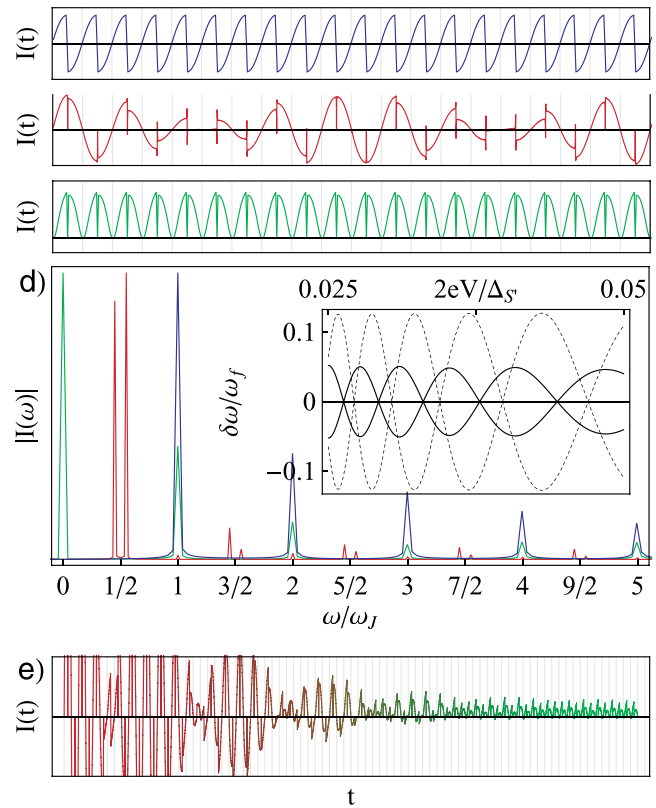

FIG. 3 (color online). Top: diagrams of the different dynamical regimes of the ac Josephson current as a function of junction bias $V$ and dimensionless conductance $G / G_{0}$, for (a) the topologically trivial and (b) nontrivial phases of a 8.7- $\mu \mathrm{m}$ long InSb wire similar to that of Fig. 2. Typical current profiles are presented in (c). Conventional adiabatic (blue, top) and multiple Andreev reflection (MAR) (green, bottom) regimes are both $2 \pi$ periodic. A wide region of beating $4 \pi$-periodic ac Josephson current (red, middle) appears in the nonadiabatic TS phase and is absent in the trivial case. Each regime has a distinct frequency transform (d). The inset shows the beat splitting $\pm \delta \omega$ of the anomalous peak at $\omega_{J} / 2$ as a function of the bias $V$, both for the even (solid) and the odd sectors (dashed). Panel (e) presents a typical dissipative decay from the $4 \pi$ regime into the stationary MAR regime, which develops a dc current component (peak at $\omega=0$ ). 
beating envelope (due to imperfect LZ transitions at $\pi, 3 \pi$, etc.). The parameter window required by this solution, $\delta_{\pi} \ll$ $2 \mathrm{eV} \ll 2 \mathrm{eV}_{\mathrm{MAR}}$, is experimentally relevant, since $\delta_{\pi}$ and $V_{\text {MAR }}$ may be independently controlled by the length $L_{S^{\prime}}$ and, e.g., the junction transparency, respectively. In contrast, in the topologically trivial phase, only the lowest of the two Andreev levels is detached from the quasicontinuum. Thus, the state $|11\rangle$ will immediately decay into $|10\rangle$ upon crossing $\phi=2 \pi$. As a consequence, the $4 \pi$-periodic current cannot develop. The anomalous $4 \pi$-periodic component in the transient regime, therefore, is an unequivocal signature of the existence of MBS in the junction. At high $V \sim V_{\mathrm{MAR}}$, the transition into the continuum becomes significant in each period $T_{2 \pi}$, for both phases. For such voltages, $t_{T} \sim T_{2 \pi}$, and close to two fermions escape into the contacts per cycle. This process yields a finite dc current [green curve (bottom) in Fig. 3(c)], which is the analogue of the multiple Andreev reflection (MAR) mechanism of conventional junctions. It should be noted that the current for any finite bias $V$ eventually develops, in the stationary regime, a finite dc component, that has the same origin as MAR, i.e., the promotion of Cooper pairs into the quasicontinuum by iterated scattering processes [see Fig. 3(e)].

A parametric diagram of the MAR (green), the $2 \pi$ adiabatic (blue), and the $4 \pi$ beating (red) regimes possible are presented in Figs. 3(a) and 3(b). The boundaries between regimes are crossovers, defined by $V=V_{\text {MAR }}$ and $2 \mathrm{eV}=\delta_{\pi}$. The different regimes may be clearly distinguished using a finite time spectral analysis of the current, shown in Fig. 3(d). The adiabatic regime exhibits components at $\omega=n \omega_{J}$, for integer $n>0$, while the MAR regime develops an additional $\omega=0$ peak. The $4 \pi$-periodic transient regime in the TS phase, on the other hand, exhibits a distinct half-integer spectrum $\omega=$ $\left(n+\frac{1}{2}\right) \omega_{J}$, together with a peak splitting due to the accompanying beating envelope. The splitting $\pm \delta \omega$ is plotted in Fig. 3(d) (inset) as a function of the applied bias, which reveals oscillations due to the interference of successive LZ processes at odd multiples of $\phi=\pi$.

Transient times. - The characteristic decay time of the transient current $I(t) \sim e^{-t / t_{T}}$ is given by $t_{T} \equiv \frac{\hbar^{2} \Gamma_{0}}{(2 \mathrm{eV})^{2}} \tau$, which scales with bias $V$ and the characteristic relaxation rate $\Gamma_{0}$ that models the escape of quasiparticles from the quasicontinuum levels into the reservoirs [23]. The dimensionless function $\tau\left(G / G_{0}\right)$ that depends solely on the junction's normal conductance is numerically computed in Fig. 4(a). It is maximum in the tunneling regime, and it decays to zero as transparency goes to one. This means that $t_{T}$ decreases when the detachment $\delta_{0} \rightarrow 0$, since then the escape probability into the quasicontinuum in each cycle goes to one. The characteristic escape voltage $V_{\text {MAR }}$ is determined from the condition $t_{T}=T_{2 \pi}, 2 \mathrm{eV}_{\mathrm{MAR}}=$ $\hbar \Gamma_{0} \tau / 2 \pi$. One can see that, while a sufficiently slow driving $V$ will suppress quasiparticle poisoning and will thus increase the duration of the transient regime, a
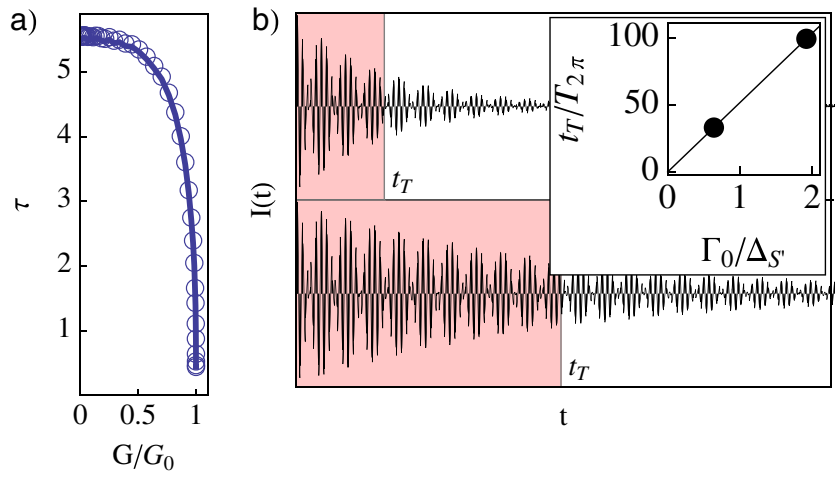

FIG. 4 (color online). Transient time scales as $t_{T} \equiv \frac{\hbar^{2} \Gamma_{0}}{(2 \mathrm{eV})^{2}} \tau$, with dimensionless function $\tau\left(G / G_{0}\right)$ plotted in (a). The transient $4 \pi$ regime [red shaded region, (b)] becomes longer as the relaxation rates to the quasicontinuum $\Gamma_{0}$ increase (Quantum Zeno effect). The two main panels in (b) show the ac Josephson current vs. time $\left(2 \mathrm{eV}=0.03 \Delta_{S^{\prime}}\right)$ and two values of $\Gamma_{0}$ highlighted in the inset, which shows the transient time $t_{T} \propto \Gamma_{0}$.

complementary, and potentially preferable, route is to engineer the environment's $\Gamma_{0}$ in order to increase the lifetime of the Majorana qubits without slowing operation. Indeed, by increasing the escape rates, parity mixing will be suppressed, increasing $t_{T}$. This is known as the quantum Zeno effect, whereby a quantum system coupled to a bath may develop longer coherence times when increasing the speed at which information is lost into the bath. In a very fast environment, the transitions into the quasicontinuum at $\phi=$ $2 \pi n$ will be suppressed by this same Zeno mechanism. This is demonstrated in the simulation of Fig. 4(b), which shows how $t_{T}$ is tripled as a result of increasing the $\Gamma_{0}$. Using realistic parameters for InSb nanowires, we estimate that typical $t_{T}$ reach into the $\mu \mathrm{s}$ range at $\mu \mathrm{V}$ bias voltages.

Owing to these long transients, the spectrum of microwave radiation should show clear features of the fractional frequencies [23]. Such measurement can be performed with an on-chip detector by using, for example, the photon assisted tunneling current of quasiparticles across a superconductor-insulator-superconductor junction capacitively coupled to the TS one. Importantly, it has been already demonstrated [36] that such technique allows a direct detection of fractional Josephson frequencies which paves the way for MBS on-chip detection [23].

We are grateful to Y.V. Nazarov, S. Frolov, L. Kouwenhoven, and S. Kohler for useful discussions. We acknowledge the support from the CSIC JAE-Doc program and the Spanish Ministry of Science and Innovation through Grants No. FIS2008-00124 and FIS2009-08744.

[1] L. Fu and C. L. Kane, Phys. Rev. Lett. 100, 096407 (2008).

[2] J. D. Sau, R. M. Lutchyn, S. Tewari, and S. Das Sarma, Phys. Rev. Lett. 104, 040502 (2010).

[3] J. Alicea, Phys. Rev. B 81, 125318 (2010). 
[4] R. M. Lutchyn, J.D. Sau, and S. Das Sarma, Phys. Rev. Lett. 105, 077001 (2010).

[5] Y. Oreg, G. Refael, and F. von Oppen, Phys. Rev. Lett. 105, 177002 (2010).

[6] C. W. J. Beenakker, arXiv:1112.1950.

[7] C. J. Bolech and E. Demler, Phys. Rev. Lett. 98, 237002 (2007).

[8] J. Nilsson, A. R. Akhmerov, and C. W. J. Beenakker, Phys. Rev. Lett. 101, 120403 (2008).

[9] K. T. Law, P. A. Lee, and T. K. Ng, Phys. Rev. Lett. 103, 237001 (2009).

[10] K. Flensberg, Phys. Rev. B 82, 180516 (2010).

[11] M. Wimmer, A. R. Akhmerov, J. P. Dahlhaus, and C. W. J. Beenakker, New J. Phys. 13, 053016 (2011).

[12] M. Gibertini, F. Taddei, M. Polini, and R. Fazio, Phys. Rev. B 85, 144525 (2012).

[13] E. Prada, P. San-Jose, and R. Aguado, arXiv:1203.4488.

[14] V. Mourik, K. Zuo, S. M. Frolov, S. R. Plissard, E. P. A. M. Bakkers, and L.P. Kouwenhoven, Science 336, 1003 (2012).

[15] A. Y. Kitaev, Phys. Usp. 44, 131 (2001).

[16] H. Kwon, K. Sengupta, and V. Yakovenko, Eur. Phys. J. B 37, 349 (2003).

[17] L. Fu and C. L. Kane, Phys. Rev. B 79, 161408 (2009).

[18] P. A. Ioselevich and M. V. Feigel'man, Phys. Rev. Lett. 106, 077003 (2011).

[19] K. T. Law and P. A. Lee, Phys. Rev. B 84, 081304 (2011).

[20] L. Jiang, D. Pekker, J. Alicea, G. Refael, Y. Oreg, and F. von Oppen, Phys. Rev. Lett. 107, 236401 (2011).

[21] For the sake of the argument, we use a fixed value $\phi=\pi$ for the zero-energy crossing although its position is not universal, see, e.g., [4].

[22] Note that a close analogy exists between Majorana and zero-energy ABS, although the latter are unconstrained by fermion parity [23].
[23] See Supplemental Material at http://link.aps.org/ supplemental/10.1103/PhysRevLett.108.257001 for a discussion about analogies with the Josephson effect through a single resonant level, details of the calculation of Andreev levels, a description of the nonequilibrium formalism, the Lindblad description of quasiparticle poisoning, and a discussion of various aspects of experimental detection.

[24] D. I. Pikulin and Y. V. Nazarov, JETP Lett. 94, 693 (2012).

[25] B. van Heck, F. Hassler, A. R. Akhmerov, and C. W. J. Beenakker, Phys. Rev. B 84, 180502 (2011).

[26] C. Wittig, J. Phys. Chem. B 109, 8428 (2005).

[27] D. M. Badiane, M. Houzet, and J. S. Meyer, Phys. Rev. Lett. 107, 177002 (2011), this recent study considers the stationary ac Josephson current in junctions of helical edge states in two-dimensional topological insulators $\left(L_{S^{\prime}} \rightarrow \infty\right)$.

[28] P. Facchi and S. Pascazio, Phys. Rev. Lett. 89, 080401 (2002).

[29] These parity states constitute an Ising qubit, $\sigma_{z}=$ $-2 i \gamma_{1} \gamma_{2}$. One can represent a full spin with three MBS [30,31] and thus a logical qubit may be constructed [32]: $\sigma_{z}=-2 i \gamma_{1} \gamma_{2} ; \sigma_{y}=-2 i \gamma_{3} \gamma_{1} ; \sigma_{x}=-2 i \gamma_{2} \gamma_{3}$.

[30] A. M. Tsvelik, Phys. Rev. Lett. 69, 2142 (1992).

[31] A. Shnirman and Y. Makhlin, Phys. Rev. Lett. 91, 207204 (2003).

[32] C. Nayak, S. Simon, A. Stern, M. Freedman, and S. Das Sarma, Rev. Mod. Phys. 80, 1083 (2008).

[33] Thus, the Hilbert space of the four MBS is that of two decoupled replicas of a logical qubit.

[34] Recently, similar ideas using a phenomenological Majorana model have been discussed in D. I. Pikulin and Y. V. Nazarov, arXiv:1112.6368.

[35] D. Averin and A. Bardas, Phys. Rev. Lett. 75, 1831 (1995).

[36] P.-M. Billangeon, F. Pierre, H. Bouchiat, and R. Deblock, Phys. Rev. Lett. 98, 216802 (2007). 